\title{
Méthodes expérimentales et numériques appliquées à la caractérisation thermophysique d'un mur en briques anciennes en conditions instationnaires
}

\section{Experimental and numerical thermophysical characterization methods applied to an old brick wall in dynamic conditions}

\author{
Emilio Sassine ${ }^{1}$, Yassine Cherif ${ }^{2}$, Emmanuel Antczak ${ }^{2}$, Joseph Dgheim ${ }^{1}$ \\ ${ }^{1}$ Laboratoire de Physique Appliquée (LPA), Université Libanaise, Faculté des Sciences, Campus Fanar, Lebanon. \\ ${ }^{2}$ Univ. Artois, ULR 4515, Laboratoire de Génie Civil et géo- Environnement (LGCgE), F-62400 Béthune, France
}

RÉSUMÉ. L'objectif de ce travail est d'améliorer les connaissances sur la caractérisation thermo-physique des murs opaques en comparant trois méthodes numériques sur un mur expérimental en maçonnerie de brique. La démarche développée ici consiste à effectuer une identification paramétrique des propriétés thermophysiques du mur considéré (à savoir la conductivité thermique $\lambda$ et la capacité thermique $\rho . c_{p}$ ), en comparant et en minimisant la différence entre les résultats des tests expérimentaux et ceux résultant du modèle numérique.

Le dispositif expérimental est constitué d'un caisson thermique à ambiance contrôlée traversé par un radiateur à bain thermostatique placé du côté opposé au mur. Le mur expérimental est un mur massif en brique $(6 \mathrm{~cm} \times 11 \mathrm{~cm} \times 22$ $\mathrm{cm}$ ) avec des joints de mortier d'une épaisseur totale de $34 \mathrm{~cm}$. II constitue l'une des quatre faces latérales du caisson.

Trois différentes méthodes ont été examinées : la méthode analytique utilisant la matrice de transfert de chaleur, la méthode des éléments finis utilisant le logiciel COMSOL $\circledast$ multiphysics, et la méthode du modèle de nœud d'air de zone (Air node zone) utilisant le Type 56 du logiciel TRNSYS ${ }^{8}$.

Les résultats obtenus sont satisfaisants pour la conductivité thermique $\lambda$ et pour la capacité thermique $\rho . c_{p}$ pour les trois méthodes étudiées.

ABSTRACT. The objective of this work is to improve knowledge on the thermo-physical characterization of building walls by comparing three numerical methods on an experimental masonry brick wall. The approach consist in a parametric identification of the thermophysical properties of the considered wall (namely the thermal conductivity $\lambda$ and the thermal capacity $\rho . c_{p}$ ), by comparing and minimizing the difference between the results of the experimental tests and those resulting from the numerical model.

The experimental device consists of a controlled atmosphere thermal box crossed by a thermostatic bath radiator placed on the side opposite the wall. The experimental wall is a solid brick wall $(6 \mathrm{~cm} \times 11 \mathrm{~cm} \times 22 \mathrm{~cm})$ with mortar joints with a total thickness of $34 \mathrm{~cm}$. It constitutes one of the four lateral faces of the box.

Three different methods were examined: the analytical method using the heat transfer matrix, the finite element method using COMSOL $\AA$ multiphysics software, and the Air node zone model method using the Type 56 of the TRNSYS® software.

The results obtained were satisfactory for $\lambda\left(0.914,0.935\right.$, and $0.884 \mathrm{~W} . \mathrm{m}^{-1} \cdot \mathrm{K}^{-1}$ and for $\rho . \mathrm{c}_{\mathrm{p}}(1091445,1057100$, and $\left.1095500 \mathrm{~J} /\left(\mathrm{m}^{3} . \mathrm{K}\right)\right)$ for the three investigated methods.

MOTS-CLÉS. Parois de bâtiment, transfert de chaleur, propriétés thermiques, méthode inverse, simulations thermiques dynamiques.

KEYWORDS. Building walls, heat transfer, thermal properties, inverse method, dynamic thermal simulation. 


\section{Nomenclature}

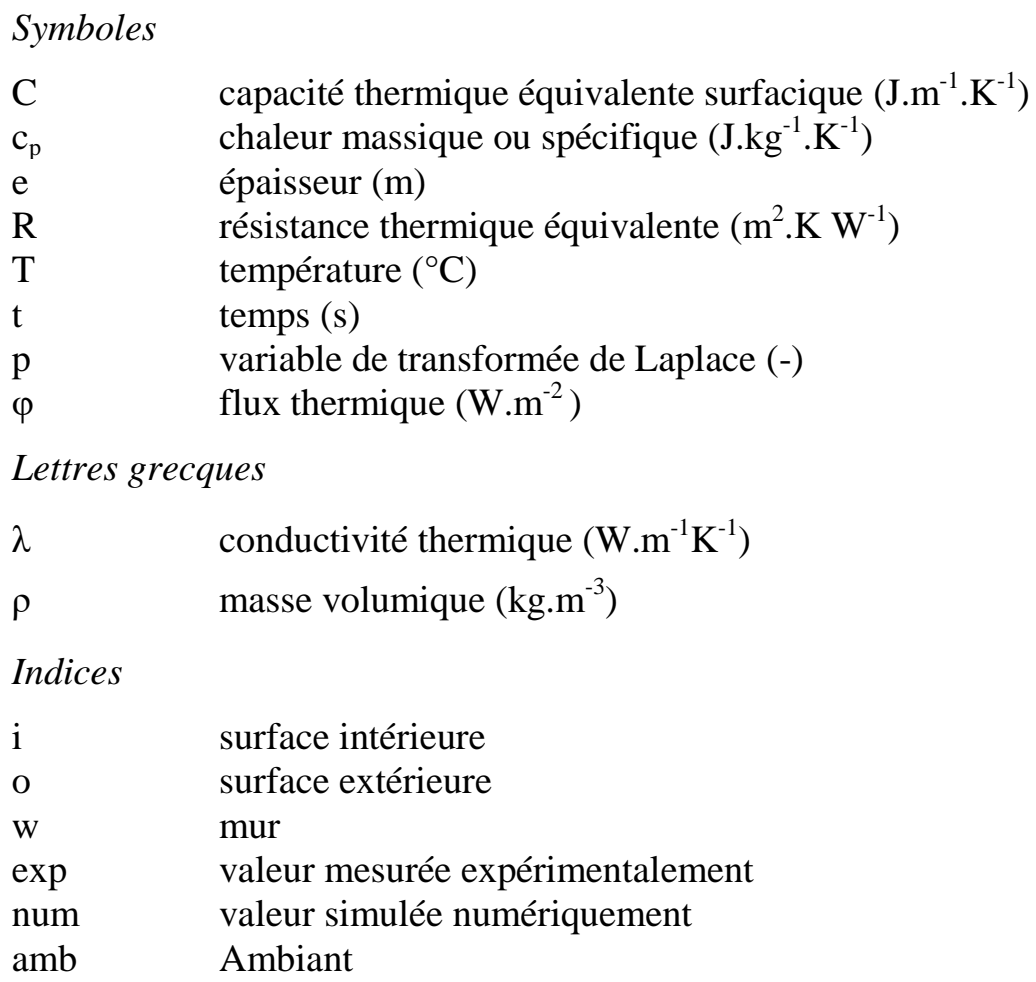

\section{Introduction}

Les problèmes énergétiques et environnementaux actuels liés à la limitation des ressources en combustibles fossiles et au réchauffement de la planète ont conduit à une focalisation croissante sur l'efficacité énergétique dans de nombreux secteurs d'énergie. L'un des principaux contributeurs au potentiel d'efficacité énergétique est le secteur du bâtiment et en particulier les enveloppes des bâtiments. Les propriétés thermophysiques de l'enveloppe du bâtiment sont considérées comme des paramètres essentiels pour évaluer et prévoir la demande en énergie des bâtiments existants ou nouveaux.

Afin de pouvoir évaluer la performance thermique globale des bâtiments et constituer un outil d'aide à la décision précieux pour la conception de bâtiments écologiques, certains attributs des bâtiments doivent être pris en compte. Ces attributs incluent la valeur U, le pouvoir absorbant des surfaces extérieures, le coefficient d'ombrage (SC) du verre, le coefficient d'ombrage externe des dispositifs d'ombrage, le coefficient de gain de chaleur solaire (SHGC) du verre, l'orientation du bâtiment et le rapport de paroi (WWR) [NAT 18].

De nombreuses formulations de transfert de chaleur utilisent des modèles thermiques en régime permanent qui ne nécessitent que des conductivités thermiques, des coefficients de transmission thermique $U$, ou bien des résistances thermiques $R$. Cette approche est encore utilisée dans de nombreuses études utilisant des dispositifs expérimentaux de laboratoire ou des mesures in situ. La méthode du thermo-fluxmètre est utilisée dans des conditions de laboratoire en régime permanent pour déterminer la conductivité thermique des matériaux de construction [BRU 18]. La norme ISO 9869 exige un capteur de flux thermique et deux thermocouples pour déterminer la résistance thermique des murs à l'aide de mesures in situ. Quelques améliorations ont été proposées par Rasooli et al. [RAS 18] ayant suggéré d'utiliser un capteur de flux de chaleur supplémentaire, opposé au premier, afin de réduire la durée des mesures et d'améliorer la précision des résultats.

Cependant, les conditions limites thermiques externes sur l'enveloppe du bâtiment ne sont pas stables en raison des conditions extérieures changeantes telles que la température, la vitesse du vent 
et le rayonnement solaire. De même, des facteurs intérieurs tels que le comportement des occupants ou l'utilisation de systèmes de chauffage ou de climatisation rendent le comportement dynamique de l'enveloppe du bâtiment de plus en plus important pour rendre l'analyse de la performance énergétique des bâtiments plus précise.

Bien que l'hypothèse de régime permanent semble être acceptable en hiver pour les régions modérées et froides, elle doit être remplacée par des conditions dynamiques en été, en particulier dans les climats chauds. Plusieurs travaux ont abordé la problématique de la détermination des propriétés thermiques des murs de bâtiment dans des conditions limites dynamiques. Les approches incluent des méthodes statistiques telles que la méthode d'inférence bayésienne, la norme EN ISO 13786 ou la solution analytique de l'équation de Fourier. Berger et al. [BER 16] ont utilisé une méthode statistique appelée inférence bayésienne pour estimer les conductivités thermiques et le coefficient de transfert de chaleur par convection interne en surveillant un mur dans un ancien bâtiment historique. La méthode semble prometteuse, mais elle ne prend en compte que la détermination de la conductivité thermique de trois matériaux composant le mur sans tenir compte des propriétés thermiques dynamiques, telles que la densité et la chaleur spécifique, qui ont été prédéterminées sur la base des normes françaises. Le modèle d'inférence bayésien a également été utilisé par Gori et al. [GOR 17] pour comparer deux modèles de masse thermique localisée bien informés (le modèle à une masse thermique $1 \mathrm{TM}$ et le modèle à deux masses thermiques $2 \mathrm{TM}$ ) ainsi que les mesures de température et de flux de chaleur sur deux murs épais de construction différente : un mur en brique pleine et un mur d'argile aérée, de plâtre, d'isolation en fibre de bois et de plaques de plâtre. La comparaison appuyait fortement le modèle 2TM sur le modèle 1TM pour décrire avec précision les données observées. Par ailleurs, la norme EN ISO 13786 a été adoptée par Petojević et al. [PET 18] pour déterminer les caractéristiques thermiques dynamiques d'un mur multicouche aux propriétés thermiques inconnues à l'aide de fonctions de réponse impulsionnelle thermique (TIR), à l'aide de données issues de mesures expérimentales in situ et d'un estimateur par les moindres carrés. Baldinelli et al. [BAL 18] ont également utilisé la norme EN ISO 13786 pour déterminer l'amplitude et le décalage temporel à travers un mur expérimenté en utilisant une boîte chaude avec des sollicitations sinusoïdales d'une période d'un jour. La méthode manque de précision avec une différence d'amplitude supérieure à $9 \%$ et se limite aux conditions aux limites sinusoïdales, ce qui la rend inadaptée aux conditions aux limites aléatoires, comme dans le cas de mesures in situ. En comparant le flux de chaleur mesuré à la solution analytique fournissant une prédiction du flux de chaleur transitoire en surface, Robinson et al. [ROB 17] ont estimé la diffusivité thermique d'un mur expérimental. Une condition limite de rampe thermique à haute température a été appliquée à une face, l'autre étant exposée à l'environnement ambiant plus froid. De la même manière, la conductivité thermique effective a été déterminée en appliquant une température de surface constante en tant que condition limite.

Les problèmes inverses font partie des problèmes les plus importants en science, car ils conduisent à des paramètres physiques qui ne peuvent pas être observés directement. Un problème inverse est le processus de calcul à partir d'un ensemble d'observations des facteurs de causalité qui les ont produites. Contrairement à un problème direct qui commence par les causes et calcule ensuite les résultats, les problèmes inverses calculent les facteurs de causalité produits par un ensemble d'observations. Bien que des problèmes inverses aient été utilisés dans diverses applications thermiques, il n'a pas été appliqué dans le contexte d'enveloppe de bâtiment pour déterminer les propriétés thermiques des murs de bâtiment. En effet, la méthode inverse a été utilisée pour déterminer la force et l'emplacement d'une source de chaleur de longueur fixe sur un mur en utilisant quelques points de données sélectionnés en aval [BAN 18], et pour reconstituer la distribution de température transitoire dans les conditions aux limites dans un élément entier sur des températures mesurées à des points choisis [DUD 16], et même pour prédire l'état du mur en briques réfractaires latérales d'un four de fusion [HAF 17]. Parmi les très rares travaux utilisés pour déterminer les propriétés thermiques des murs en utilisant la méthode inverse, on trouve les travaux 
de Chaffar et al. [CHA 14] qui utilisaient une surface de résistance chauffante plate de $0,9 \mathrm{~m} \times 1,1$ $\mathrm{m}$ appuyée contre le mur testé par un panneau de polystyrène de $18 \mathrm{~cm}$ d'épaisseur afin de diriger la plus grande partie de la puissance dissipée par la résistance dans le mur testé. Le procédé consiste à examiner thermiquement un mur en appliquant un flux de chaleur et à étudier la réponse en termes de température enregistrée par thermographie infrarouge sur la surface opposée. Bien que la méthode donne des résultats satisfaisants, elle peut difficilement être utilisée pour les mesures sur site car elle nécessite l'utilisation d'une source plane transitoire qui n'est pas facilement transportable. A retirer des mesures in situ sont réalisées.

L'équipe Habitat de LGCgE travaille depuis plusieurs années à la caractérisation numérique et expérimentale de différents matériaux de construction. Ces méthodes reposent généralement sur des mesures directes de la température et du flux de chaleur appliqués dans des conditions expérimentales en laboratoire ou in-situ. Les approches développées, que ce soit dans le domaine fréquentiel par impédance thermique ou bien par des méthodes inverses dans le domaine temporel, visent à proposer des méthodes efficaces pour la détermination des propriétés thermophysiques des parois.

Cet article explore trois méthodes différentes permettant l'identification paramétrique des propriétés thermophysiques $\left(\lambda\right.$ et $\left.\rho . c_{p}\right)$ des murs. Ces trois méthodes ont été appliquées à un cas expérimental de mur en maçonnerie de briques et les résultats obtenus sont très satisfaisants pour les trois méthodes. Elles sont applicables dans des conditions climatiques réelles et sur des murs existants, offrant ainsi la possibilité de déterminer les propriétés thermiques dynamiques équivalentes des parois in-situ.

\section{Description générale du problème et dispositif expérimental :}

\subsection{Dispositif expérimental}

Le mur expérimental étudié est un mur en briques pleines semblable aux murs des maisons anciennes du nord de la France; il est constitué de briques pleines de $(6 \mathrm{~cm} \times 11 \mathrm{~cm}$ x $22 \mathrm{~cm})$ assemblées par des joints de mortier et d'une épaisseur totale de $34 \mathrm{~cm}$ [SAS 17a, SAS 16, SAS 17b, SAS 17c, SAS 19].

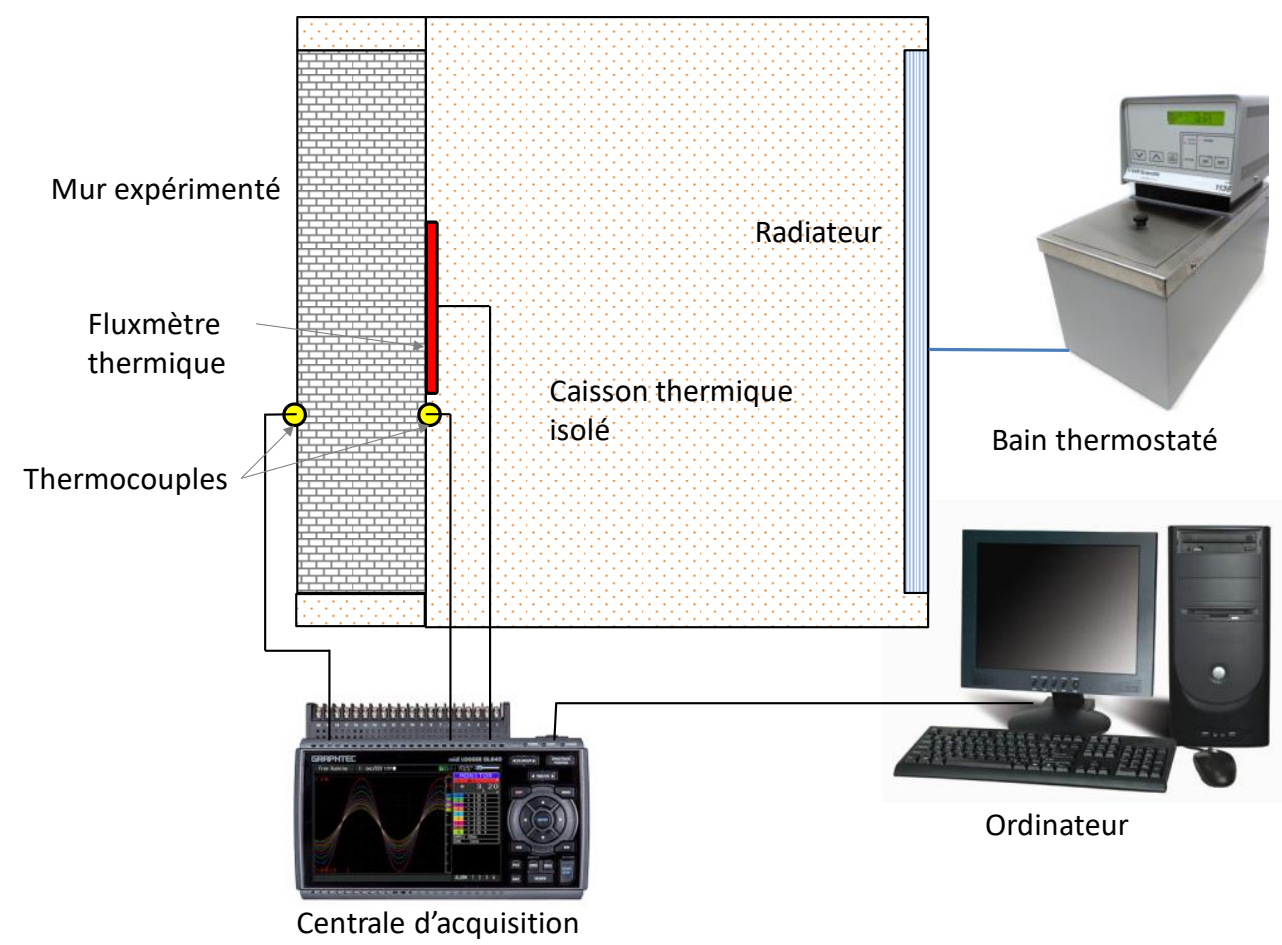

Figure 1. Dispositif expérimental 
Le mur est soumis à une ambiance contrôlée grâce à une boîte chauffante isotherme contenant un radiateur dont la température du fluide, contrôlée par un bain thermostaté régulé à $+/-0.1^{\circ} \mathrm{C}$, varie entre $5^{\circ} \mathrm{C}$ et $60^{\circ} \mathrm{C}$.

Les surfaces latérales du mur ont été isolées par $20 \mathrm{~cm}$ de laine de roche, ce qui permet de réduire le transfert de chaleur à un flux de chaleur unidimensionnel. La boîte chauffante a également été fortement isolée pour réduire les pertes de chaleur.

La Fig. 1 schématise le dispositif expérimental (mur et caisson thermique) en coupe transversale avec ses composants principaux.

Deux thermocouples (type T) donnent les températures intérieure et extérieure de la paroi, et un fluxmètre $\left(15 \times 15 \mathrm{~cm}^{2}\right)$ mesure le flux traversant la paroi à la surface intérieure de la boîte de chauffage. Le fluxmètre a été placé au centre du mur de sorte à couvrir un volume élémentaire représentatif de brique et de mortier comme le montre la Fig. 2b; ceci permettra d'éviter le problème dû à la présence de deux matériaux différents (blocs de maçonnerie et joints de mortier). Tous les capteurs sont connectés à une centrale d'acquisition de données qui enregistre les données avec des pas de temps réguliers de $0,5 \mathrm{~h}$.

\subsection{Hypothèses : mur homogène et transfert de chaleur unidirectionnel}

Le mur de maçonnerie composé de blocs de brique pleine et de joints de mortier est supposé être un mur homogène ayant une conductivité thermique équivalente $\lambda$ et une capacité thermique équivalente $\rho . c_{p}$. Le flux de chaleur est supposé être unidirectionnel car les bords latéraux du mur sont très bien isolés. Le problème est ainsi réduit à un transfert de chaleur unidirectionnel à travers une paroi équivalente homogène ayant les propriétés thermiques équivalentes $\lambda$ et $\rho . c_{p}$.

\subsection{Conditions aux limites}

Le profil de température du fluide caloporteur circulant dans le radiateur est prédéfini par un ensemble de valeurs générées par le programme LABVIEW. Il impose dans la boîte de chauffage une température $T_{i}$, sur la face intérieure du mur, une température $T_{w i}$ et, sur la face extérieure du mur, une température $T_{w o}$. Ces valeurs ont été utilisées comme conditions aux limites dans le modèle numérique.

$\varphi_{i}$ et $\varphi_{o}$ correspondent respectivement aux flux de chaleur interne et externe aux limites des parois interne et externe.

Dans l'étude expérimentale (Fig. 2a), l'intérieur de la boîte représente l'environnement extérieur de la maison, qui varie de manière aléatoire, et l'atmosphère du laboratoire (en dehors de la boîte) représente l'ambiance intérieure de la maison où la température varie légèrement autour de $22^{\circ} \mathrm{C}$, comme indiqué sur la Fig. 3.

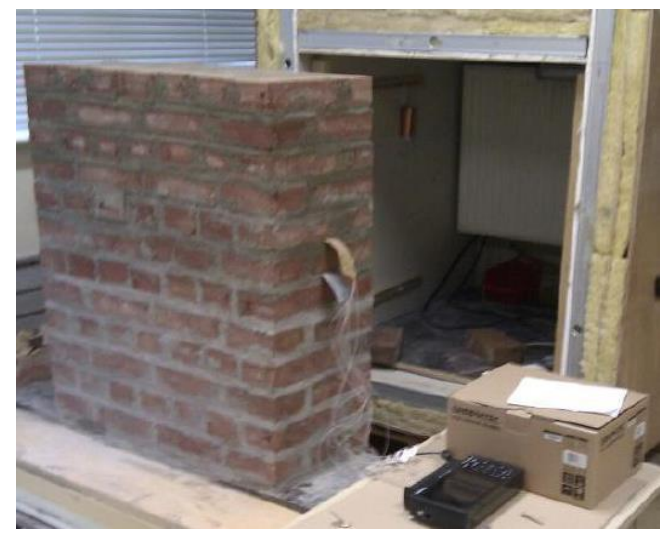

(a)

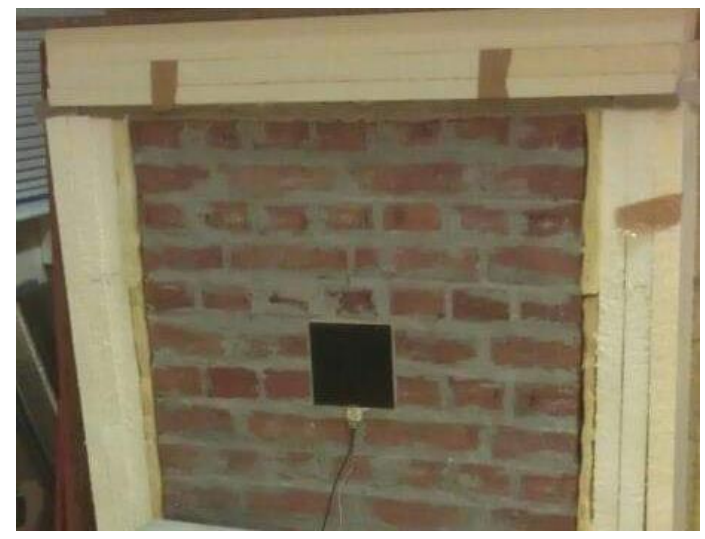

(b)

Figure 2. Dispositif expérimental (a) et emplacement du fluxmètre (b) 
Le fluide caloporteur impose dans le caisson thermique une température $T_{i}(t)$ qui chauffe la face de la paroi interne pour atteindre une température $T_{w i}(t)$ par transfert de chaleur par convection, cette chaleur traverse la paroi par conduction et crée à la surface de la paroi externe une température $T_{w o}(t)$. La température de l'air ambiant dans le laboratoire a la valeur $T_{o}(t)$. Les quatre profils de températures $T_{i}(t), T_{w i}(t), T_{w o}(t)$ et $T_{o}(t)$. Les données de température et de flux de chaleur ont été enregistrées à des intervalles de temps réguliers (30 minutes), comme illustré à la Fig. 3.

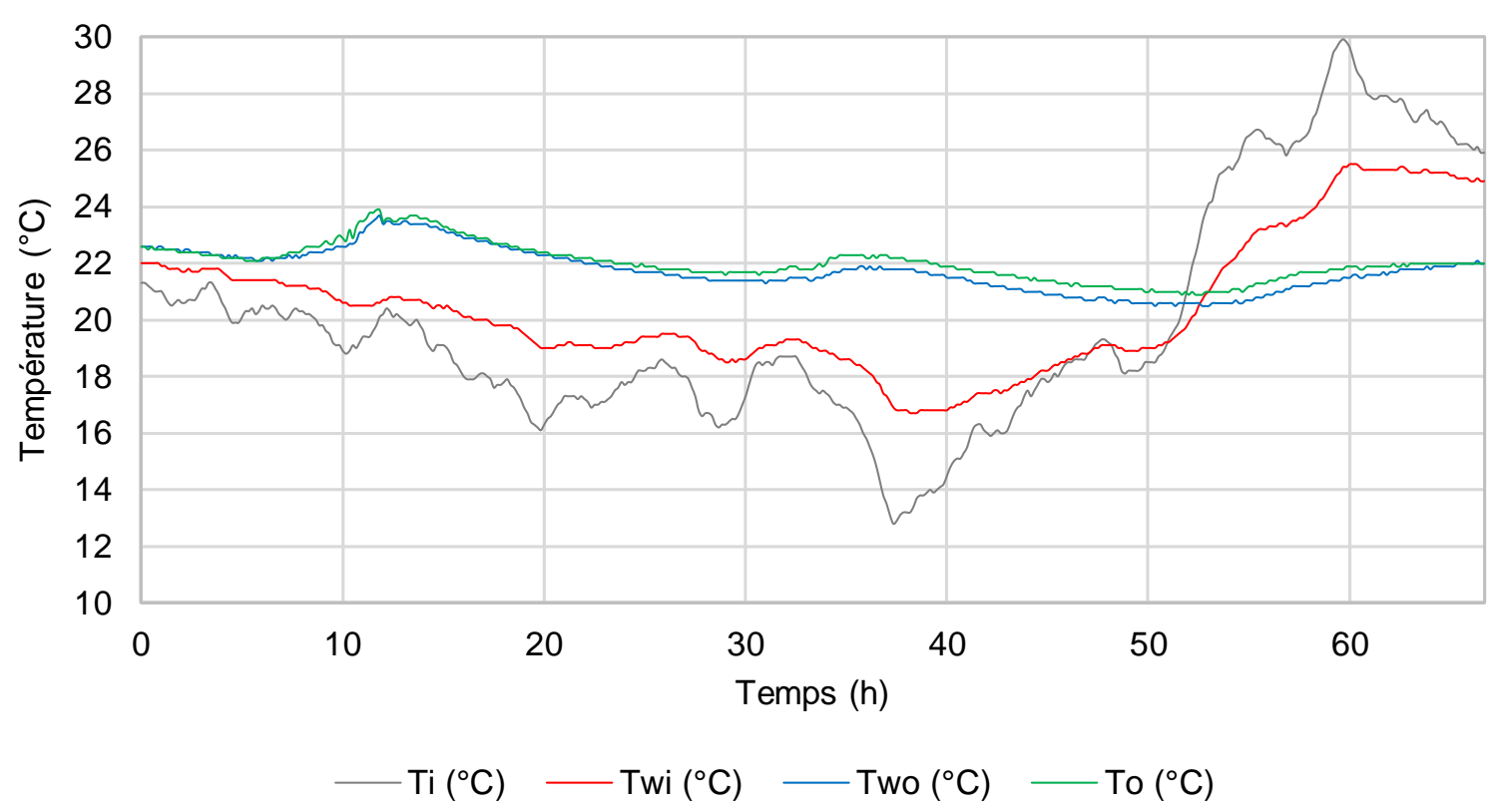

Figure 3. Conditions aux limites appliquées au dispositif expérimental

\section{Description des méthodes numériques étudiées}

\subsection{Description du problème physique}

Dans les problèmes de transfert de chaleur directs, les paramètres $R$ et $C$ sont connus et les conditions aux limites (températures et / ou flux de chaleur) sont les inconnues à déterminer.

Connaissant les températures de part et d'autre du mur à travers les mesures de $T_{i}$ et $T_{0}$, le problème de méthode inverse se ramène à un problème de conduction en régime dynamique.

Les propriétés thermiques spécifiques au mur expérimenté ont été déterminées en utilisant comme conditions limites les températures imposées sur les faces interne $\left(T_{w i}\right)$ et externe $\left(T_{w o}\right)$ du mur et en minimisant la différence entre le flux de chaleur mesuré sur la face interne du mur $\varphi_{i \_ \text {exp }}$ et le flux thermique obtenu numériquement sur la même face $\varphi_{i \_n u m}$ pour la fonction objectif $\varphi_{i}$. Dans ce cas, deux variables de contrôle ont été optimisées simultanément grâce à des algorithmes d'optimisation inverses utilisés dans les différentes méthodes pour déterminer la conductivité thermique $\lambda$ et la capacité thermique $\rho . c_{p}$.

La résistance thermique $R$ et la capacité thermique surfacique $C$ sont liées à la conductivité thermique $\lambda$ et la capacité thermique volumique $\rho . c_{p}$ par :

$$
\begin{aligned}
R & =\frac{e}{\lambda} \\
C & =\rho \cdot C_{p} . e
\end{aligned}
$$

Dans la Fig. 4, la méthode proposée par l'organigramme suggère des enregistrements réguliers toutes les 30 minutes et un calcul d'optimisation réalisé toutes les 5 heures (10 valeurs) en 
comparant le flux théorique $\varphi_{\text {theo }}$ au flux expérimental $\varphi_{\text {exp }}$ afin de déduire les valeurs optimales de $R$ et de $C$ permettant de minimiser l'écart entre ces deux flux. L'acquisition des données s'arrête quand 6 valeurs consécutives d'optimisation de $\mathrm{R}$ et $\mathrm{C}$ convergent (erreur $<\varepsilon$ ).

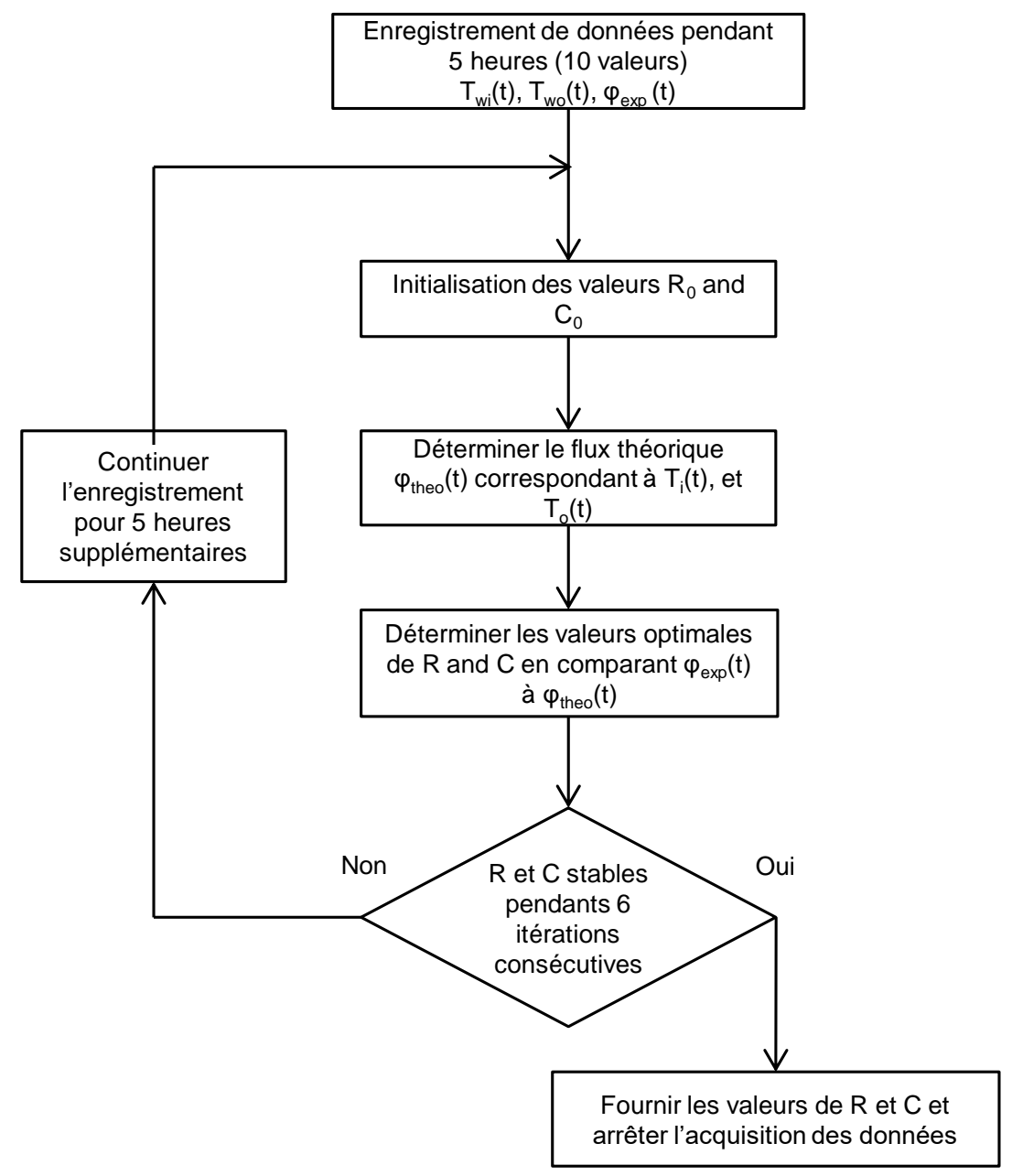

Figure 4. Schéma de principe pour la méthode de caractérisation proposée

Trois méthodes d'optimisation différentes seront confrontées :

1. La méthode analytique utilisant la matrice de transfert de chaleur

2. La méthode des éléments finis utilisant le logiciel COMSOL multiphysics

3. La méthode du modèle de nœud d'air de zone (Air node zone) utilisant le Type 56 du logiciel TRNSYS.

\subsection{Méthode analytique}

Selon la norme EN ISO 13786, l'équation de transfert en fonction de la variable p de Laplace et de la relation entre les transformées de Laplace des températures $\left(\theta_{w o}\right.$ et $\left.\theta_{w i}\right)$ et des flux de chaleur $\left(\Phi_{w o}\right.$ et $\left.\Phi_{w i}\right)$ des deux côtés d'un mur homogène peut être exprimée sous la forme de fonctions hyperboliques :

$$
\left[\begin{array}{l}
\theta_{w o} \\
\Phi_{w o}
\end{array}\right]=\left[\begin{array}{cc}
\operatorname{ch}(\sqrt{p R C}) & \sqrt{\frac{R}{p C}} \operatorname{sh}(\sqrt{p R C}) \\
\sqrt{\frac{p C}{R}} \operatorname{sh}(\sqrt{p R C}) & \operatorname{ch}(\sqrt{p R C})
\end{array}\right]=\left[\begin{array}{l}
\theta_{w i} \\
\Phi_{w i}
\end{array}\right]
$$


Le processus d'optimisation est réalisé par la méthode inverse [SAS 16] en comparant des données expérimentales avec le modèle théorique en utilisant la méthode des moindres carrés.

Après la détermination du flux thermique théorique pour les valeurs initiales de $R_{0}$ et $C_{0}$, la méthode des moindres carrés est utilisée pour minimiser la somme des écarts entre le flux thermique mesuré et calculé, afin de trouver les valeurs optimales de $\mathrm{R}$ et $\mathrm{C}$. L'optimisation est réalisée à l'aide de l'algorithme GRG2 (Gradient Réduit Généralisé) qui est normalement utilisé pour optimiser les problèmes non linéaires.

\subsection{Méthode des éléments finis}

COMSOL $®$ Multiphysics est un logiciel multiplateforme d'analyse par éléments finis permettant de simuler des problèmes multi physiques ; il offre des interfaces utilisateur classiques basées sur la physique et des systèmes couplés d'équations aux dérivées partielles (EDP). Lors de la résolution des modèles, COMSOL Multiphysics ${ }^{\circledR}$ utilise la méthode des éléments finis (FEM). Un ensemble d'équations caractéristiques (décrivant les propriétés physiques et les conditions aux limites) est attribué à chaque élément, qui est ensuite résolu en tant qu'ensemble d'équations simultanées permettant de prédire le comportement de l'objet.

Le modèle de transfert de chaleur dans les solides utilise la version de l'équation de chaleur de l'Eq. (3) comme modèle mathématique pour le transfert de chaleur dans les solides :

$$
\rho C_{p} \frac{\partial T}{\partial t}-\nabla \cdot(k \nabla T)=Q
$$

$\rho$ est la masse volumique du matériau, $\mathrm{C}_{\mathrm{p}}$ la capacité calorifique, $\mathrm{k}$ la conductivité thermique et $\mathrm{Q}$ la source de chaleur.

Les paramètres thermophysiques de la paroi $\left(\lambda\right.$ et $\left.\rho . c_{p}\right)$ ont été optimisés en utilisant l'algorithme de Levenberg - Marquardt (LMA). L'algorithme LMA est normalement utilisé pour résoudre les problèmes de moindres carrés non linéaires et est utilisé dans de nombreuses applications logicielles pour résoudre les problèmes d'ajustement de courbe des moindres carrés. Il a été choisi pour sa rapidité de simulation et sa fiabilité [SAS 19].

\subsection{Nœud d'air de zone}

Le logiciel TRNSYS ${ }^{\circledR}$ avec son composant TRNBuild ${ }^{\circledR}$ permet de modéliser le comportement thermique d'un bâtiment divisé en différentes zones thermiques. Le programme TRNBUILD $®$ lit et traite un fichier contenant la description du bâtiment et génère deux fichiers qui seront utilisés par le composant TYPE 56 au cours d'une simulation TRNSYS ${ }^{(\text {Fig. 5). }}$

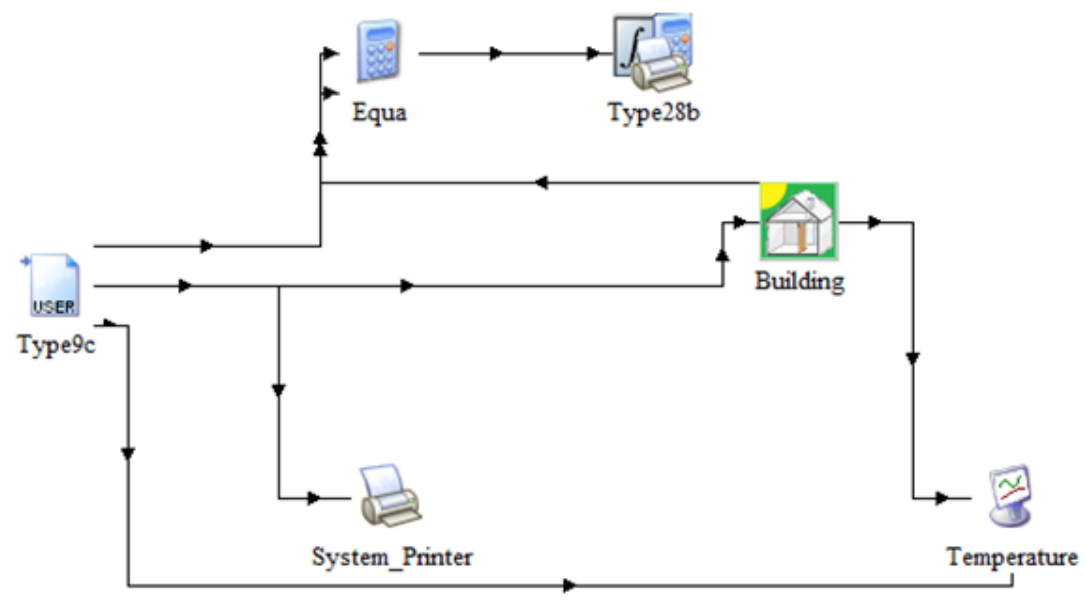

Figure 5. Schéma bloc du modèle (Type 56) utilisé 
Le fichier contenant la description du bâtiment traitée par TRNBUILD® peut être généré par l'utilisateur avec n'importe quel éditeur de texte ou avec le programme interactif TRNBUILD®. Le modèle de bâtiment de TYPE 56 est un modèle de balance non géométrique avec un nœud d'air par zone (Fig. 6), représentant la capacité thermique du volume d'air de la zone et des capacités étroitement liées au nœud d'air (meubles, par exemple).

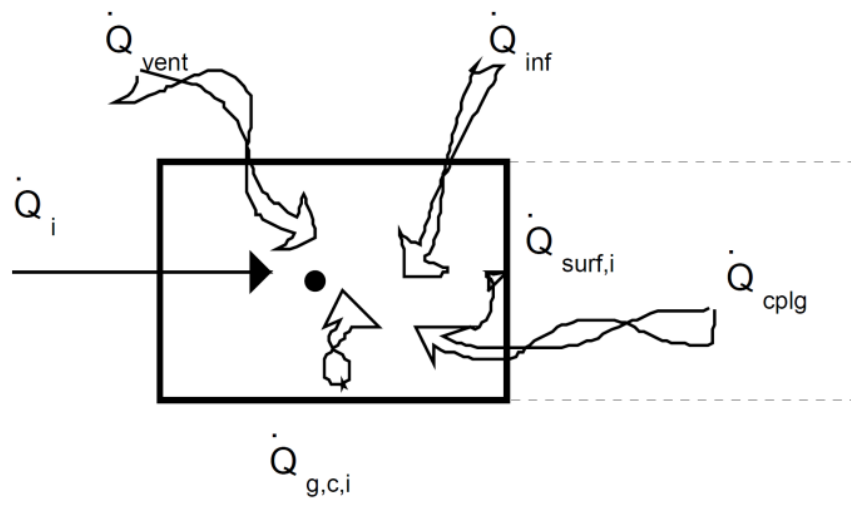

Figure 6. Bilan thermique sur le nœud d'air de la zone

Les calculs de transfert de chaleur de TRNSYS sont basés sur la méthodologie de la fonction de transfert introduite par Stephenson et Mitalas [STE 71].

L'optimisation a été effectuée avec GenOpt®, un logiciel d'optimisation permettant de minimiser une fonction objectif évaluée par un programme de simulation externe (dans cette étude TRNSYS). Il utilise les algorithmes génétiques permettant d'obtenir une solution approchée à un problème d'optimisation en utilisant la notion de sélection naturelle et l'appliquant à une population de solutions potentielles au problème donné. Les variables indépendantes peuvent être des variables continues (éventuellement avec des limites inférieures et supérieures), des variables discrètes ou les deux. Les contraintes sur les variables dépendantes peuvent être mises en œuvre à l'aide de fonctions de pénalité ou de barrière.

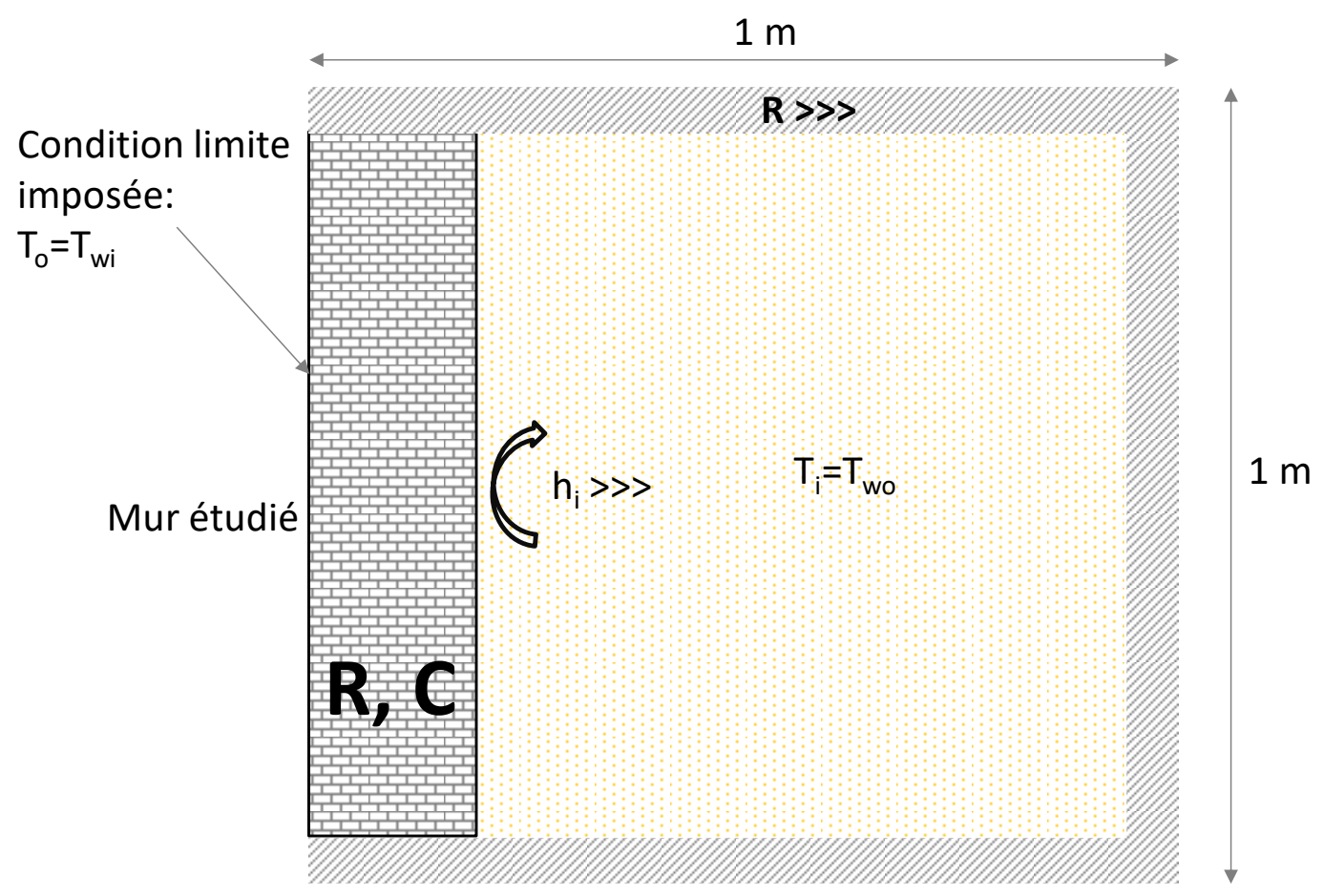

Figure 7. Conditions aux limites imposes sur le mur étudié 
Les conditions aux limites sont imposées sur une des parois du mur dans l'interface TRNBuild du logiciel TRNSYS, les autres parois étant adiabatiques (résistances thermiques extrêmement élevées).

Comme indiqué dans la Fig. 7 a paroi est soumise d'une part à une condition limite de température extérieure imposée $\mathrm{T}_{0}=\mathrm{T}_{\mathrm{wi}}$, et d'autre part à une condition limite d'ambiance imposée $\mathrm{T}_{\mathrm{i}}=\mathrm{T}_{\text {wo }}$ avec un coefficient d'échange convectif intérieur extrêmement élevé n'étant pas possible d'imposer une température directement sur le mur du côté intérieur à travers le logiciel.

Les trois données analysées sont :

1. La température de surface intérieure du mur «TSI»

2. La température de surface intérieure du mur «TSO»

3. Et l'énergie transférée au niveau de la surface extérieure «QCOMO »

Cette dernière représente la fonction-objectif qui sera minimisée en la comparant au flux expérimental mesure au niveau de la surface intérieure du mur (du côté du caisson) $\varphi_{\mathrm{i}}$.

\section{Résultats et discussion}

\subsection{Résultats des trois méthodes étudiées}

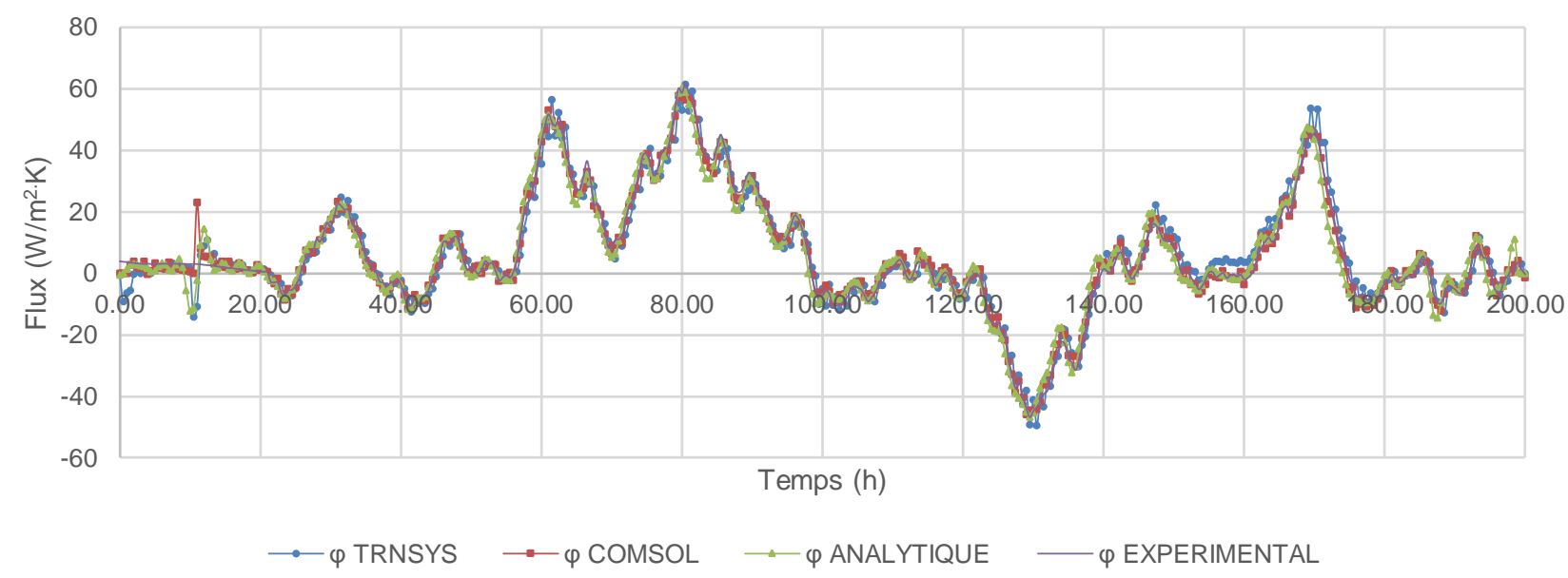

Figure 8. Comparaison des flux numériques pour les solutions optimales des trois méthodes avec le flux expérimental mesuré

Les trois méthodes numériques utilisées offrent des résultats clairement comparables entre eux et avec les résultats expérimentaux. Ceci est visiblement clair en comparant les courbes de variation du flux thermique pour les valeurs optimales de $\lambda$ et $\rho . c_{p}$ (Fig. 8). Le temps total de simulation considéré est de 200 heures (un peu plus que 8 jours). 


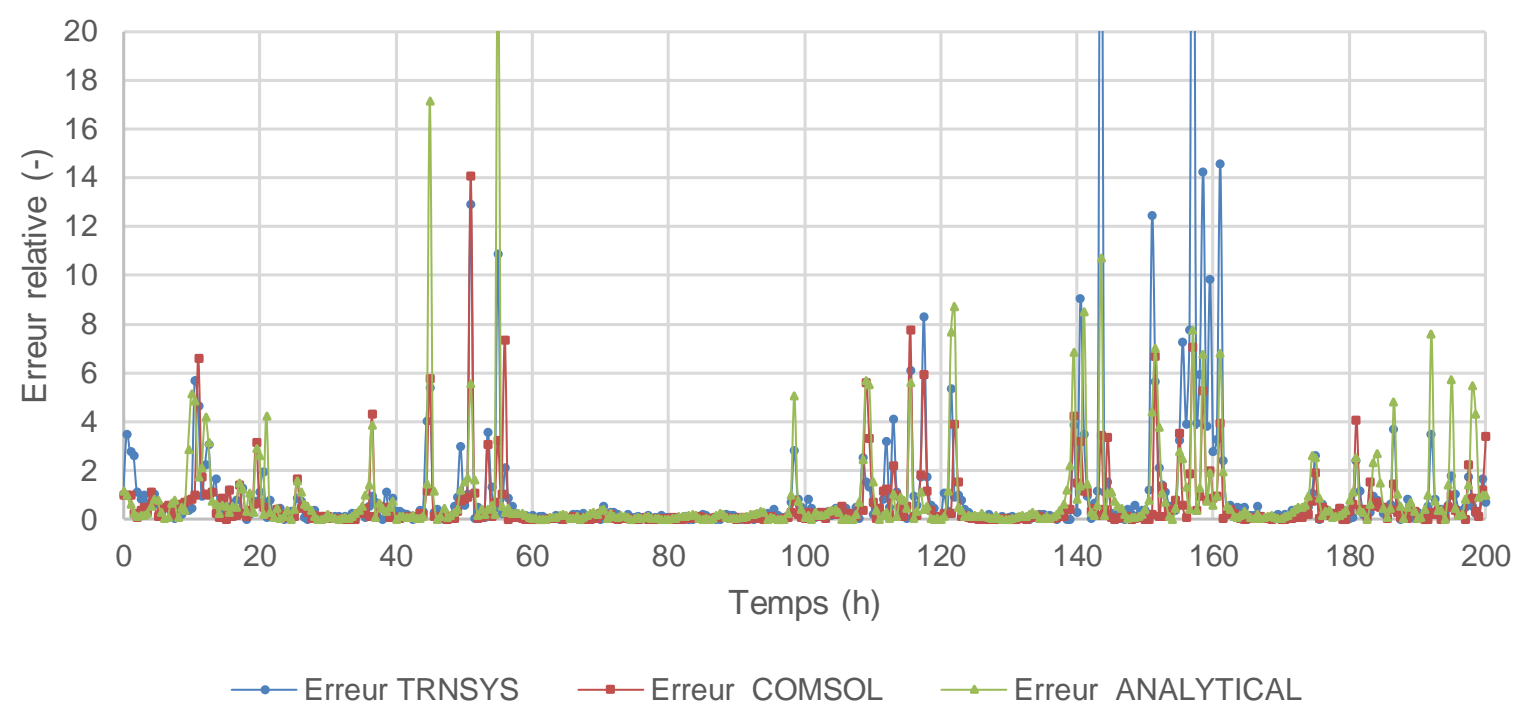

Figure 9. Comparaison des erreurs relatives entre les flux numériques et le flux expérimental mesuré pour les solutions optimales des trois méthodes

La Fig. 9 montre l'évolution de l'erreur relative pour les différentes valeurs (400 valeurs mesurées sur 200 heures avec un pas de temps de 30 minutes). Les valeurs d'erreurs sont généralement faibles et augmentent quand les valeurs de flux sont faibles et voisines de 0 (pour $t \sim 50, t \sim 100$, et $t \sim 150$ ), ce qui est très attendu puisque les erreurs augmentent quand les valeurs mesurées sont faibles. L'analyse de ces données dans le tableau 1 montre le nombre de valeurs dont l'erreur relative est inférieure à une valeur donnée $(0.1,0.2,0.3$ et 1$)$ parmi les 400 valeurs simulées sur les 200 heures pour les valeurs optimales de $\lambda$ et $\rho . c_{p}$ et pour les trois méthodes étudiées. Ces données montrent que la méthode TRNSYS et la méthode analytique offrent des résultats légèrement plus précis que la méthode COMSOL. Des études de cas supplémentaires seront nécessaires pour généraliser ce constat.

\begin{tabular}{|c|c|c|c|}
\hline & Méthode TRNSYS & Méthode COMSOL & Méthode ANALYTIQUE \\
\hline Erreur $<0.1$ & 107 valeurs & 166 valeurs & 97 valeurs \\
\hline Erreur $<0.2$ & 166 valeurs & 230 valeurs & 176 valeurs \\
\hline Erreur $<0.3$ & 213 valeurs & 266 valeurs & 218 valeurs \\
\hline Erreur $<1$ & 325 valeurs & 346 valeurs & 320 valeurs \\
\hline
\end{tabular}

Tableau 1. Evaluation de la précision des trois méthodes

La convergence des solutions est aussi très claire pour les logiciels d'optimisation LevenbergMarquardt (Fig. 10) et GenOpt (Fig. 11). Le temps de calcul pour la méthode analytique est très faible aux alentours de $15 \mathrm{~s}$; cependant pour les méthodes numériques les durées sont $3 \mathrm{~min} 22 \mathrm{~s}$ pour COMSOL et $5 \mathrm{~min} 55 \mathrm{~s}$ pour TRNSYS. 


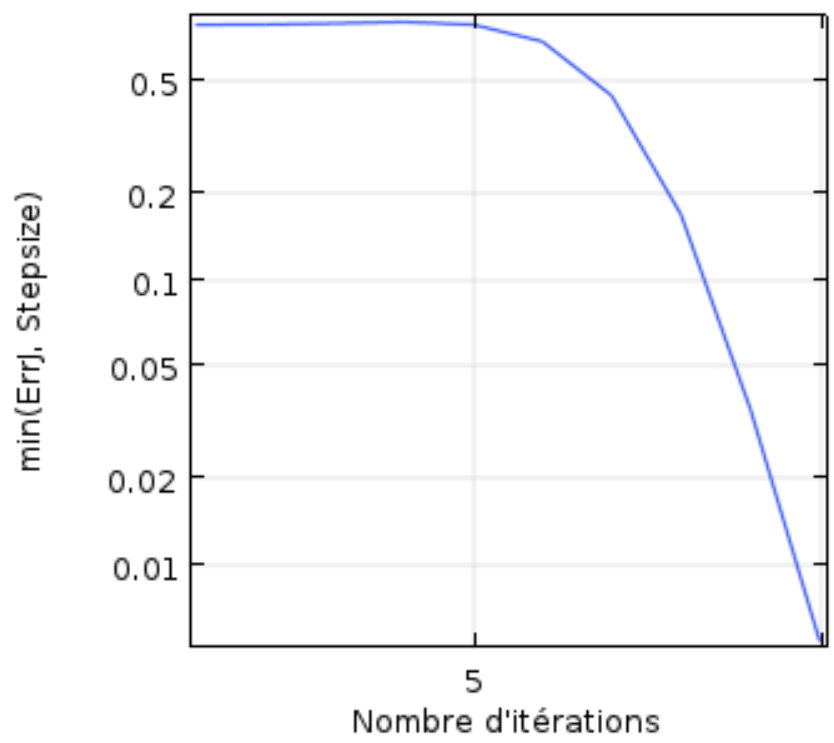

Figure 10. Graphique de convergence pour l'optimisation Levenberg-Marquardt

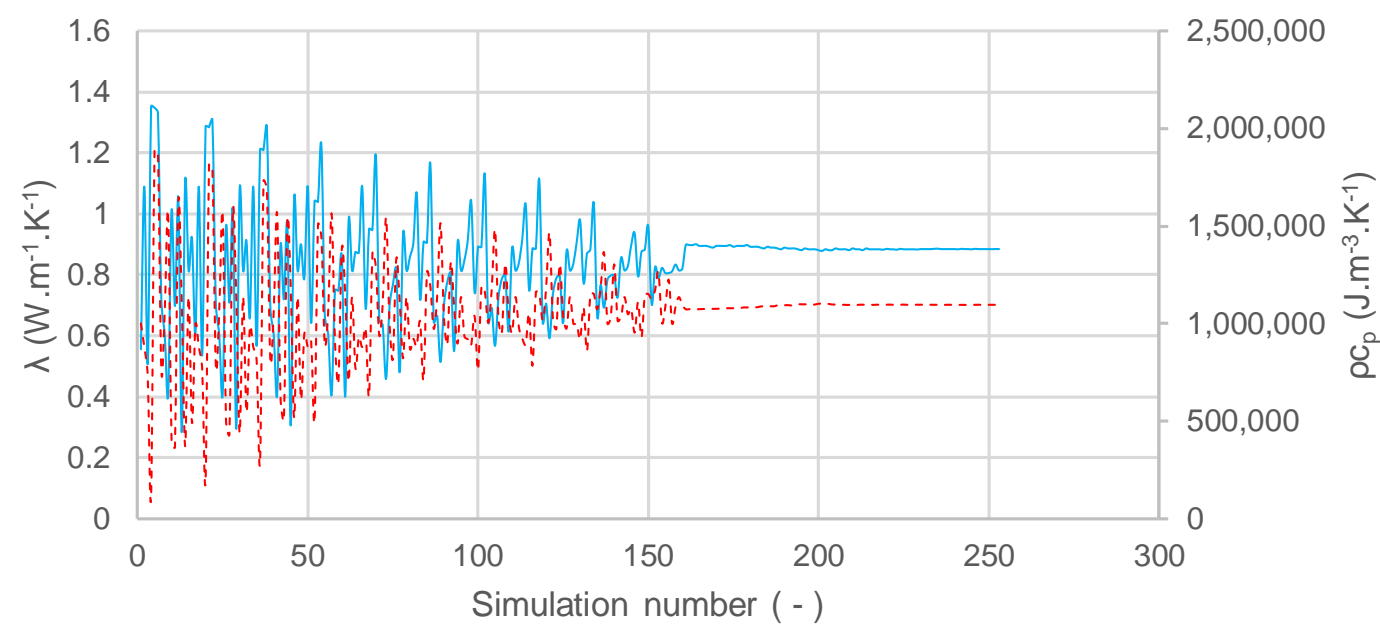

Figure 11. Graphique de convergence pour l'optimisation GenOpt

\subsection{Comparaison des résultats}

La confrontation des résultats de conductivité thermique $\lambda$ (Fig. 12) et de capacité thermique $\rho . c_{p}$ (Fig. 13), montre clairement la validité des trois méthodes et la précision dans les résultats d'identification avec un écart de $5,5 \%$ pour $\lambda$ et $3,5 \%$ pour $\rho . c_{p}$. 


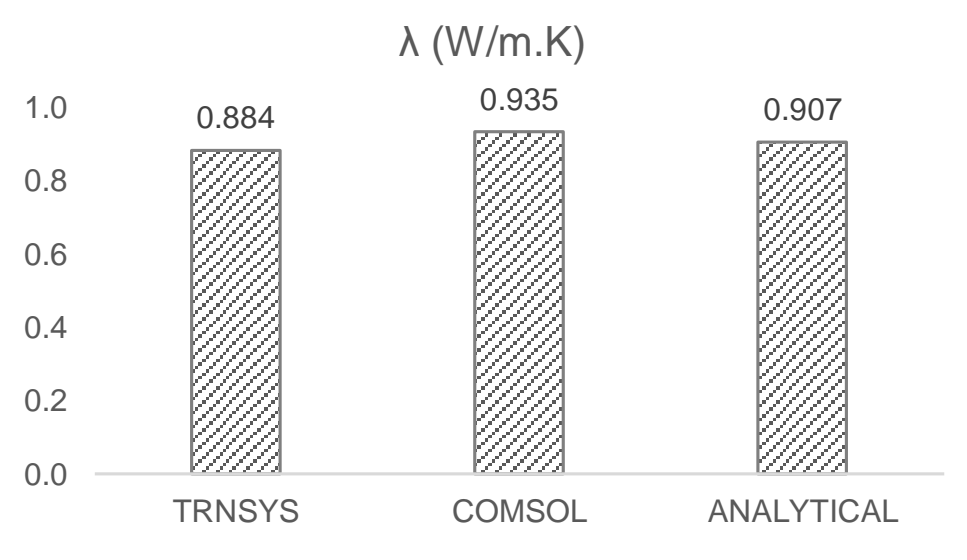

Figure 12. Comparaison entre les résultats de conductivité thermique pour les trois méthodes utilisées

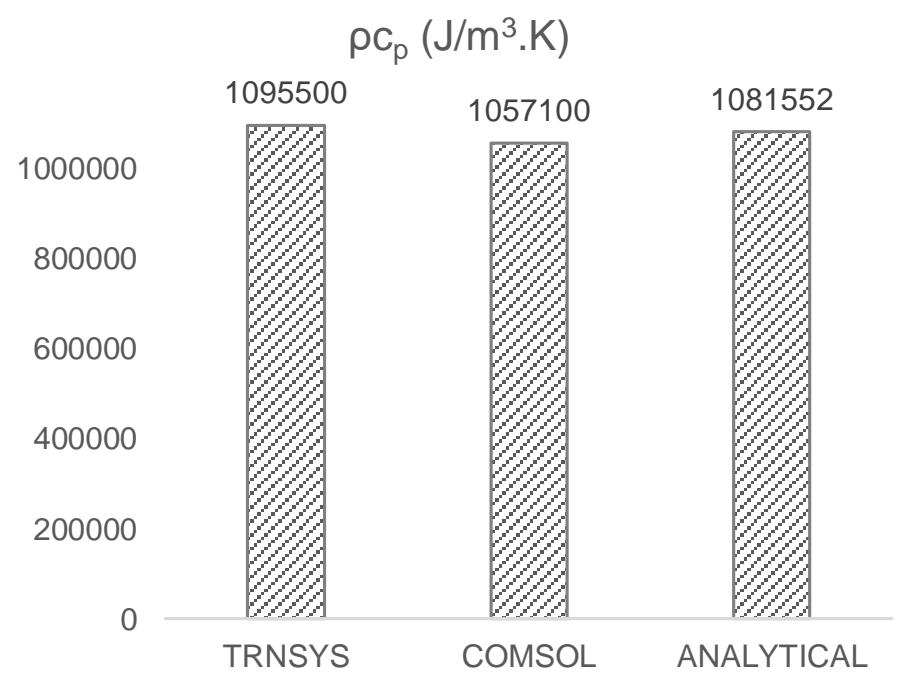

Figure 13. Comparaison entre les résultats de capacité thermique pour les trois méthodes utilisées

\section{Conclusion et perspectives}

Ce travail met en évidence trois méthodes numériques différentes permettant la caractérisation thermique des parois existantes à travers des essais simples non destructifs basés sur la mesure des températures des parois de part et d'autre ainsi que l'un des flux thermiques (de préférence le flux extérieur). La comparaison des trois méthodes confirme leur applicabilité et donne des valeurs très voisines à la fois pour la conductivité thermique $\lambda\left(5,5 \%\right.$ d'écart) et pour la capacité thermique $\rho . c_{p}$ (3,5\% d'écart).

Ces trois méthodes sont alors applicables et pourraient être utilisées pour divers types de parois existantes avec des dispositifs expérimentaux relativement simples et des périodes de mesures acceptables.

Cette démarche d'optimisation automatique des paramètres thermophysiques $(R$ et $C)$ à partir des mesures des températures intérieures et extérieures du mur $\left(T_{w i}\right.$ et $\left.T_{w o}\right)$ et du flux thermique extérieur $\varphi_{\text {exp-wo }}$ a permis de comparer l'efficacité des trois méthodes décrites et la précision des résultats.

Ces modèles présentés pour le mur en brique en question sont valables et applicables pour différents types de parois existantes. Dans les perspectives, nous exploiterons les méthodes développées sur des murs existants multicouches. 


\section{Références}

[NAT 18] W. NATEPHRA, N. YABUKI, T. FUKUDA, "Optimizing the evaluation of building envelope design for thermal performance using a BIM-based overall thermal transfer value calculation”, Building and Environment 136 (2018) $128-145$

[BRU 18] R. Bruno, P. Bevilacqua, G. Cuconati, N. Arcuri, "An innovative compact facility for the measurement of the thermal properties of building materials : first experimental results", Applied Thermal Engineering 143 (2018) 947-954

[RAS 18] A. RASOOLI, L. ITARD, "In-situ characterization of walls' thermal resistance: An extension to the ISO 9869 standard method", Energy \& Buildings 179 (2018) 374-383

[BER 16] J. BERGER, H. ORLANDE, N. MENDES, S. GuERnOUTI, "Bayesian inference for estimating thermal properties of a historic building wall”, Building and Environment 106 (2016) 327-339

[GOR 17] V. GORI, V. MARINCIONI, P. BIDDULPH, C. ELWELL, "Inferring the thermal resistance and effective thermal mass distribution of a wall from in situ measurements to characterize heat transfer at both the interior and exterior surfaces", Energy and Buildings 135 (2017) 398-409

[PET 18] Z. PETOJEVIĆ, R. GosPaviĆ, G. TODOROVIĆ, "Estimation of thermal impulse response of a multi-layer building wall through in-situ experimental measurements in a dynamic regime with applications", Applied Energy 228 (2018) 468-486

[BAL 18] G. BALdinelli, F. BiAnChI, A. LeChOwsKa, J. SChNOTALE, "Dynamic thermal properties of building components: Hot box experimental assessment under different solicitations", Energy \& Buildings 168 (2018) 1-8

[ROB 17] A.J.Robinson, F.J.LESAGE, A.ReILly, G.MCGRANAGHAN, G.BYRNe, R.O'HEGARTY, O.KInNANE, “A new transient method for determining thermal properties of wall sections", Energy and Buildings 142 (2017) 139-146

[BAN 18] A. BANGIAN-TABRIZI, Y. JALURIA, “An optimization strategy for the inverse solution of a convection heat transfer problem", International Journal of Heat and Mass Transfer 124 (2018) 1147-1155

[DUD 16] P. DUDA, "A general method for solving transient multidimensional inverse heat transfer problems", International Journal of Heat and Mass Transfer 93 (2016) 665-673

[HAF 17] M. HAFID, M. LACROIX, "Inverse heat transfer prediction of the state of the brick wall of a melting furnace", Applied Thermal Engineering 110 (2017) 265-274

[CHA 14] K. ChAFFAR, A. CHAUCHOIS, D. DEFER, ET L. ZALEWSKI, "Thermal characterization of homogeneous walls using inverse method", Energy and Buildings 78 (2014) 248-255

[SAS 17a] E. SASSINE, Z. YOUNSI, Y. CHERIF, A. CHAUCHOIS, E. ANTCZAK, "Experimental determination of thermal properties of brick wall for existing construction in the north of France", Journal of Building Engineering 14 (2017) 15-23

[SAS 16] E. SASSINE, "A practical method for in-situ thermal characterization of walls", Case Studies in Thermal Engineering 8 (2016) 84-93

[SAS 17b] E. SASSINE, Z. YOUNSI, Y. CHERIF, E. ANTCZAK, "Thermal performance evaluation of a massive brick wall under real weather conditions via the Conduction Transfer function method", Case Studies in Construction Materials 7 (2017) 56-65

[SAS 17c] E. SASSINE, Z. YOUNSI, Y. CHERIF, E. ANTCZAK, "Frequency domain regression method to predict thermal behavior of brick wall of existing buildings", Applied Thermal Engineering 114 (2017) 24-35

[SAS 19] E. SASSINE, Y. CHERIF, E. ANTCZAK, "Parametric identification of thermophysical properties in masonry walls of buildings", Journal of Building Engineering vol. 25100801 84-93 (2019)

[STE 71] STEPHENSON, MiTALAS, "Calculation of heat conduction transfer functions for multilayer slabs", ASHRAE Trans. 77 (1971) 117-126 